\title{
Nonstationary Time Series Analysis applied to Investigation of Brainstem System Dynamics
}

\author{
Ralf Vandenhouten, Manfred Lambertz, Peter Langhorst, and Reinhard Grebe* Member, IEEE
}

\begin{abstract}
Previous investigations of the dynamic organization of the lower brainstem and its relation to peripheral and other central nervous sub-systems were predominantly performed by linear methods. These are based on time-averaging algorithms, which merely can be applied to stationary signal intervals. Thus the current concept of the common brainstem system (CBS) in the reticular formation (RF) of the lower brainstem and basic types of its functional organization have been developed. Here we present experiments where neuronal activities of the RF and the nucleus tractus solitarii (NTS, first relay station of baroreceptor afferents) were recorded together with related parameters of EEG, respiration, and cardiovascular system. The RF neurons are part of the CBS, which participates in regulation and coordination of cardiovascular, respiratory, and motor systems, and vigilance. The physiological time series thus acquired yield information about the internal dynamic coordination of the participating regulation processes. The major problem in evaluating these data is the non-linearity and instationarity of the signals. We used a set of especially designed time resolving methods to evaluate non-linear dynamic couplings in the interaction between CBS neurons and cardiovascular signals, respiration and the EEG, and between NTS neurons (influenced by baroreceptor afferents) and CBS neurons.
\end{abstract}

Index terms-instationarity, time series analysis, physiological signals, brainstem system

\section{INTRODUCTION}

Living systems are exposed to permanent changing of internal and external conditions. So it is a prerequisite for their sufficient and successful functioning that internal organisms processes are able to adapt to these changes and to tune to each other. To achieve this, the organism has the capability to initiate or stop, to accelerate or decelerate individual processes of subsystems.

From this, it follows that an organism must be observed as a complex, dynamic system with predominantly non-linear and instationary properties. In life sciences, it has become a point of major interest how such continuous adaptations operate and how they are achieved. Physiological signals can provide information about global and

R. Vandenhouten is with Datan GmbH, Teltow M. Lambertz is with Inst. Physiology, FU Berlin P. Langhorst is with Inst. Physiology FU Berlin *R.Grebe is with GBM, UMR CNRS 6600, UTC Compiegne partial processes in the organism and their coordination. Especially in times of systems adaptation they are characterized by transients, phase transitions, or persistent instationarities. These properties can be investigated by analytical methods, which do not require stationarity or permit the pursuit of instationarities, respectively. Timeresolving procedures are the appropriate tools, since they do not characterize time series by static quantities but by time-dependent functions instead. In this paper, several algorithms for multiple time series analysis based on the theory of dynamic systems, statistics and time-frequency analysis are applied to biosignals representing the complex couplings of physiological subsystems. In physiological experiments, neuronal activities of the reticular formation (RF) of the lower brainstem and of the nucleus tractus solitarii (NTS) were recorded together with related parameters of EEG, respiration, and the cardiovascular system.

The RF neurons are part of a common brainstem system (CBS) for basic regulation and coordination of several functional systems of the organism. They exert influences on e.g. respiration, heart, vessels, motor systems and vigilance thus realizing behavior patterns [1,17,34]. Furthermore they receive afferent influences from somatosensory receptors, visceral receptors - cardiovascular and respiratory systems - and from higher brain structures $[27,31,40]$, i.e. the CBS has manifold feedback loops. The complex anatomical and functional organization of the RF has already been described in detail as well as interconnections with other brain areas $(1,17,31,34,35,39)$. Some of the important interrelationships of the CBS neurons in the RF are given in the scheme of Fig. 1. Results concerning the different types of dynamic organization of neurons in the CBS network have been published in detail $(4,18,19,23,26,27,28,29,40,41)$.

Activity levels of the peripheral vegetative and somatic systems and of the CNS are adapted to behavior patterns as regulated by the CBS neurons. From the numerous afferent inflows to the CBS the baroreceptor afferents are the most important inhibitory ones. Baroreceptor activation in the carotid sinus or in the aortic arch leads to an activation of neurons in the NTS [20,30], which is the first relay station for baroreceptor afferents in the CNS. From here CBS neurons are inhibited $[21,26,30]$. This inhibition induces a decrease in sympathetic activity, blood pressure, 


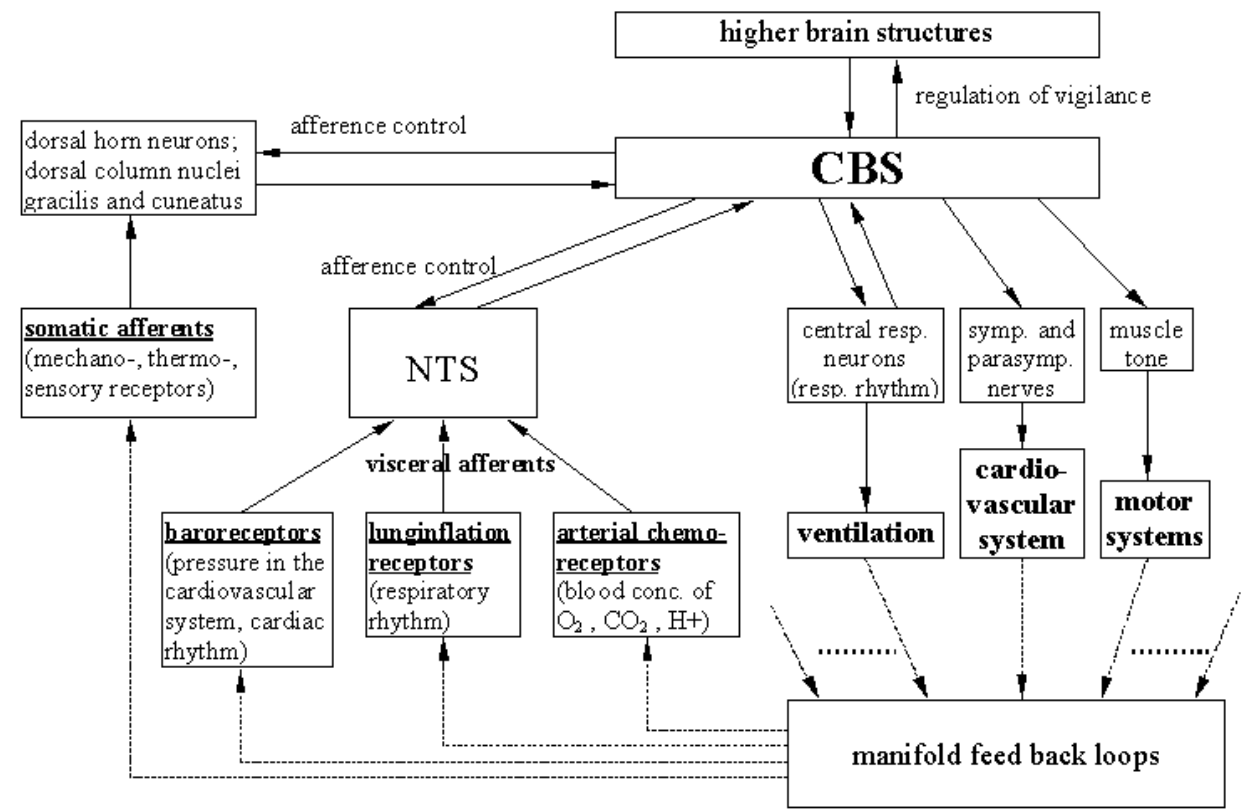

Fig. 1. Scheme of some important relationships between reticular formation neurons of the common brainstem system (CBS) and other functional systems of the organism (NTS: nucleus tractus solitarii).

and heart rate. At the same time, respiration, muscle tone and vigilance are reduced also [27], sleep can be induced [14]. This is a general activitydampening effect that can initiate and maintain the so-called trophotropic functional organization $[11,26,31]$ of the organism. The opposite effect is attained by activation of arterial chemoreceptors, which activate the CBS neurons. This leads to excitation in vegetative and somatomotor systems and in the CNS. Sleep can be interrupted and chemoreceptor activation induces ergotropic behavioral patterns [11,26,31]. The actual functional organization of neuronal structures is then characterized not only by the absolute activity level, but also by specific discharge patterns of the CBS neurons [26,27,40,41].

The above already mentioned Neurons of the dorsomedial part of the NTS receive further afferents from other visceral receptor, e.g. pulmonary receptors and chemoreceptors. In addition, they are influenced by neurons of other CNS structures, e.g. the CBS, hypothalamus, or the amygdala complex. All these inputs together influence the processing of baroreceptor afferent signals by these NTS neurons [20,30,43,44].

Our concept of the dynamic organization of the CBS neurons under influence of actually effective afferents [26,27,31,40,41], and of processing and transfer of afferent signals is described in former publications [21,22,23]. Temporarily occurring rhythms in neuronal activities are characteristic for distinct changes in functional organization of CBS neurons. The discharging of CBS neurons exhibit rhythms of different time ranges as mainly expressed in respiratory, in EEG delta-theta band and cardiac activity [21,23,24,25,26,27,28,29].
These rhythms occur as well in subsystems influenced by the CBS, e.g. in efferent sympathetic and parasympathetic nerves, in motoneurons and in neuronal activity of higher brain structures, e.g. the amygdala complex [21,22,23]. Certain links between cardiovascular and respiratory rhythms have been shown $[9,10,12,15,16,23,28,29,31,36,37$, 43,44]. Appearance and disappearance of such rhythms as well as the change in phase relations of 'relative coordination' are expression of continuously altering coordination of subsystems of the organism. This was shown by a study published recently [23], which is based on the attentive analysis of the activity of 598 brainstem neurons and their relation to peripheral systems.

We will show how suitable methods can be used for the analysis of this kind of instationary dynamics and of phase transitions appearing due to changing interrelationship of subsystems. The results of the present paper fit very well to former ones obtained from numerous experiments evaluated by other analytical methods $[4,20-31,40,41,43,44]$.

\section{S IGNAL PROCESSING}

\section{A. Recurrence Plots for testing non-stationarity}

Some of the computations described in this section need the phase space embedding of the signals. This was done according to Takens' theorem [45] using the embedding dimension $\mathrm{m}$ and the lag $\tau$. $\tau$ is determined by the position of the first minimum of the first derivative of the signals' autocorrelation

Reinhard Grebe, Dépt. GBM, UMR CNRS 6600, Université de Technologie de Compiègne 
function, and $\mathrm{m}$ is determined using the False Nearest Neighbors algorithm [21,43].

In analyzing dynamic systems so-called Recurrence Plots (RPs) introduced by Eckmann et al. [6] are most suitable for the detection of non-stationarities. This method generates graphical point patterns to give a visual demonstration of stationarity breaks and other dynamic properties of a time series. The $\mathrm{RP}$ consist of $\mathrm{N} \times \mathrm{N}$ matrix of points each of which can be either set (black) or not set (white). For setting a point $(\mathrm{i}, \mathrm{j})$ in this matrix a radius $r$ must be selected (usually $0.05 \leq \mathrm{r} \leq 0.2$ ) for the search neighborhood in the phase space as well as the maximum number $M$ (usually $10 \leq M \leq 50$ ) of considered nearest neighbors. As the coordinates $(i, j)$ represent points in time, the RP provides information about the temporal correlation of phase space points. In stationary signals these should not depend on the distance $i-j$, and consequently the texture of the plot as a whole should (at least on a rough scale) appear homogenous. In contrast, inhomogeneities occur in the texture of RPs of nonstationary signals. An aggregation of points close to the main diagonal indicates, e.g., continuous instationarities. A sequence of rectangular blocks of increased point density, on the other hand, represents a phase transition between different modes of operation (e.g. two attractors). If the texture is homogeneous within such a block, a stationary behavior can be assumed for the corresponding sub-period.

Thus, RPs prove to be a useful tool for the preprocessing of experimental time series, and provide a comprehensive image of the dynamic course at a glance.

\section{B. Time-frequency distributions and scalograms}

The aim of a time-dependent spectral representation is to indicate the intensity $P(t, \omega)$ of a certain frequency $\omega$ in a signal $x(t)$ at a given time $t$. This is called a time-frequency distribution (TFD), the most well known of which is the Wigner-Ville distribution $[2,46]$.

Even so a useful tool for time-dependent spectral analysis, it exhibits some disadvantages as e.g. occasional negative values [3]. To overcome this we apply a window function $h(\tau)$. Furthermore we scale the window function with the frequency to get a fix relation $\Delta \omega / \omega$ instead of the frequency uncertainty $\Delta \omega$. This can be achieved, i.e. by replacing $h(t)$ by $\sqrt{|\omega|} h(\omega \tau)$ :

$$
\begin{aligned}
& P_{x}(t, \grave{u})=\left|\int d \tau x(\tau) e^{-i \tau \omega} \sqrt{|\grave{u}|} h(\grave{u}(\tau-t))\right|^{2} \\
& =\mid\left\langle x, \emptyset^{*},\right\rangle^{2} .
\end{aligned}
$$

This is called a scalogram, which can be taken as the absolute square of a wavelet transform. Wavelets of this kind are called Morlet Wavelets [33]. The computational expense of this procedure is proportional to the length of the time series and the number of sample points in the frequency domain. Concerning its frequency-dependent time resolution, the scalogram is superior to the WignerVille distribution as well as to the conventional spectrogram. Compared to ARMA spectra it is more robust [47].

\section{Instantaneous Phase}

If an oscillating system has a clear (although possibly fluctuating) dominant frequency and a sufficiently narrow band spectrum the concept of an instantaneous frequency and an instantaneous phase $^{1}(I P)$ can be well defined [38], i.e. the signal $\xi(t)$ can be represented as

$$
\xi(t)=\operatorname{Re}\left[A(t) e^{i \phi(t)}\right]=\operatorname{Re}[w(t)] .
$$

$\phi(t)$ is the instantaneous phase and $d \phi(t) / d t$ the instantaneous frequency [7]. With respect to the preservation of the system dynamics the calculation of the analytical signal $w(t)$ can be performed by the Hilbert transform $\eta=\mathbf{H}(\xi)$ [47], which delivers

$$
\begin{array}{rc}
w(t)=\xi(t)+i \eta(t) & \text { and } \\
\phi(t)=\arctan \frac{\eta(t)}{\xi(t)}
\end{array}
$$

An appropriate bandpass filtering is necessary as preprocessing. Furthermore, the phase $\phi$ is not a continuous function because the arctan is "wrapped" into the interval from 0 to $2 \pi$. This problem can be solved with an unwrapping algorithm based on a continuity criterion as postprocessing. A further application of the IP is the use for detection of phase or frequency synchronization of two signals by calculating the Instantaneous Phase Difference (IPD)

$$
\Delta \phi(t)=\phi_{1}(t)-\phi_{2}(t)
$$

or the Instantaneous Frequency Ratio (IFR)

$$
I F R=\frac{\left[d \phi_{1}(t) / d t\right]}{\left[d \phi_{2}(t) / d t\right]},
$$

respectively. This permits a direct visualization of the phase relation. E.g., horizontal plateaus in the time course of the IPD $\Delta \phi(t)$ indicate the occurrence of phase locking.

\section{Coupling measures}

In order to investigate the temporal dynamics of the adaptation processes more closely we apply as coupling measures the Pointwise Transinformation (PTI) and the Pointwise Conditional Coupling Divergence (PCCD) [47]. The PTI is defined according the Transinformation I $\tau$ ) [8] for two

\footnotetext{
${ }^{1}$ The expression «phase» here describes a property of the signal's course in time and must not be confused with the expression «phase» being part of «phase space» or «phase transition» describing the state of a system.
} 
observable quantities $\xi$ and $\chi$ as derived from Shannon's information concept [42]. We use an estimation of the phase space densities by the calculation of empirical point densities $P_{i}(r)$. The $P_{i}(r)$ are determined within balls of the radius $r$ around the points $\boldsymbol{x}_{\mathrm{i}}$ of the reconstructed trajectory in the phase space. Thus, we obtain the PTI for the $i$-th time step, lag $\tau$, and the neighborhood with the radius $r$ by

$$
P T I(\tau, r, i)=\log \frac{P_{i}^{\xi \chi}(\tau, r)}{P_{i}^{\xi}(r) P_{i}^{\chi}(r)}
$$

The increase or decrease of the mean PTI level over the time gives information about changes in the coupling behavior of the investigated systems. The time resolution of the PTI is principally limited merely by the sampling rate. Thus, one is able to pursue also very fast changes in the coupling behavior, which is not possible with conventional methods (e.g. short time cross correlation) [47].

However, only static attractor properties are taken into account and that normalization (e.g. to a measure between 0 and $100 \%$ ) is difficult. In addition, there is no direct analytic relation to the coupling strength of the interacting subsystems. That is why we introduced as a dynamic measure for the coupling of two subsystems the Conditional Coupling Divergence (CCD). Like the PTI it makes use of a simple phase space reconstruction.

Two points $\left(\xi_{i}, \chi_{i}\right)$ and $\left(\xi_{j}, \chi_{j}\right)$ of coupled systems lying close together in the common phase space should still be neighbors after a short period $t_{e}=l \cdot t_{a}$. Therefore the CCD is defined as the expectation of the conditional probability for one point located in a neighborhood of radius $r$ around a reference trajectory in the common phase space at time $t_{0}$ being still in this neighborhood after $l$ time steps [47], i.e.

$$
\begin{aligned}
& C C D(r, l)=\left\langlep \left[\left(\xi_{j+l}, \chi_{j+l}\right)\right.\right. \\
& \left.\left.\in B_{r}\left(\xi_{i+l}, \chi_{i+l}\right) \mid\left(\xi_{j}, \chi_{j}\right) \in B_{r}\left(\xi_{i}, \chi_{i}\right)\right]\right\rangle
\end{aligned}
$$

where $B_{r}\left(z_{0}\right):=\left\{z_{0} ;\left\|z-z_{0}\right\|<r\right\}$.

Thus, the $C C D$ is normalized to values between 0 and 1. Applying the ergodic theorem [5] the CCD can be calculated empirically, similar to the transinformation, as an average of local point densities in the reconstructed phase space, i.e.

$$
C C D(r, l)=\frac{1}{N} \sum_{i} \operatorname{PCCD}(r, l, i)
$$

with Pointwise CCD (PCCD)

$$
\begin{aligned}
& \operatorname{PCCD}(r, l, i)= \\
& \frac{\sum_{j=0}^{N-1} \theta\left(r-\left\|\Delta_{j, i}\right\|\right) * \theta\left(r-\left\|\Delta_{j+l, i+l}\right\|\right)}{\sum_{k=0}^{N-1} \theta\left(r-\left\|\Delta_{k, i}\right\|\right)}
\end{aligned}
$$

where $\Delta_{\mathrm{j}, \mathrm{i}}=\left(\mathrm{x}_{\mathrm{j}}, \mathrm{y}_{\mathrm{j}}\right)-\left(\mathrm{x}_{\mathrm{i}}, \mathrm{y}_{\mathrm{i}}\right)$ and $\theta$ is the Heaviside function. Since the numerical calculation requires only simple arithmetic and logical operations, it can be implemented efficiently.

Compared to other phase space measures the PCCD is relatively robust against changes of the control parameter $r$ because the scaling behavior of the attractors is of minor importance.

\section{METHODS}

\section{A. Experiments}

The methods used in the animal experiments are described in detail a previous publication [23]. In short: Experiments were performed in adult mongrel dogs anesthetized with chloraloseurethane. The dogs were intubated and breathed room air spontaneously. The medulla oblongata was exposed from the dorsal side, musculature removed layer by layer and the squama occipitalis removed osteoclastically. The cerebellum remained, the exposed medulla was covered by mineral oil at body temperature. Brainstem neurons were recorded extracellularly with glass-insulated platinum electrodes of $10 \mu \mathrm{m}$ diameter riding freely in the tissue, which avoids artifacts due to movements of the brain.

Two RF neurons were recorded in the actual experiment via one electrode $(1.1 \mathrm{~mm}$ rostral and $2.15 \mathrm{~mm}$ right lateral to the obex) and a NTS neuron by another one $(0.15 \mathrm{~mm}$ rostral and $1.3 \mathrm{~mm}$ left lateral to the obex). In addition, measures of blood pressure (via a catheter in the aorta), intrapleural pressure (IPP) as a measure of respiration, ECG, parieto-occipital EEGs left and right, and efferent renal sympathetic nerve activity (symp) were taken.

\section{B. Processing of the physiological signals}

The recorded signals were stored on polygraph and magnetic tape. Neuronal spikes and R-waves of the ECG were converted into sequences of rectangular pulses by means of window discriminators (shapes and amplitudes of the neuronal spikes were watched by superposition on storage oscilloscopes, using electronic delay circuits). This procedure provides separated sequences of rectangular pulses for the different neuronal activities and the ECG. They were digitized and stored on computer as event trains (accuracy $0.1 \mathrm{~ms}$ ). From these event trains, equidistant time series of the instantaneous heart frequency and the instantaneous discharge frequencies of the neurons were computed.

Before digitizing (sample rate $1 \mathrm{kHz}$ ) the signals were filtered to avoid aliasing (stop band above $500 \mathrm{~Hz}, \leq-60 \mathrm{~dB})$. The EEGs were filtered (0.1$30 \mathrm{~Hz}$ ), the renal sympathetic activity was full wave rectified and filtered $(0-15 \mathrm{~Hz})$. For sample rate reduction (to $100 \mathrm{~Hz}$ ) all data were digitally low pass filtered (FIR, 50Hz). From the arterial blood 


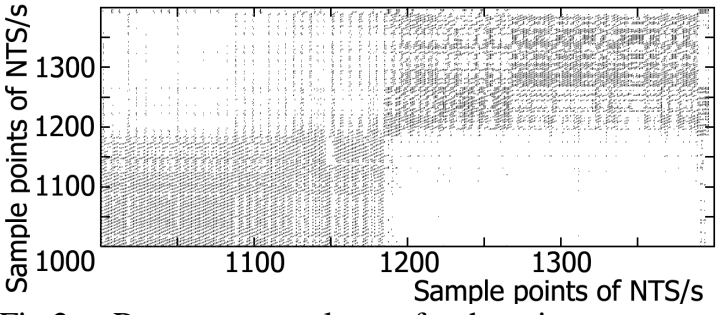

Fig.2: Recurrence plot of the instantaneous discharge frequency of the NTS neuron, which was computed from the event trains as described in III.B. A phase transition is to be seen at $1180 \mathrm{~s}$ (parameters: $\mathrm{m}=5, \mathrm{M}=50, \mathrm{r}=0.18$ ).

pressure the envelopes of systolic maxima and diastolic minima were computed by interpolation. By FIR-filtering, EEGs were split into three frequency bands, $0.6-2 \mathrm{~Hz}$ (» $\delta$-band»), $2.5-7 \mathrm{~Hz}$

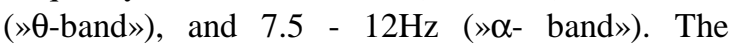
steepness of the FIR-filter rolloff was $40 \mathrm{~dB} / 0.5 \mathrm{~Hz}$, so there was no overlapping of frequency bands.

From these signals envelopes of their minima and maxima were computed, and afterwards the differences $» \Delta »$ of these envelopes resulted in time series of $\Delta(\gg \delta$-band $\gg), \Delta(\gg \theta$-band $\gg)$, and $\Delta(\gg \alpha-$ band»). For the analysis of the EEGs only these $\Delta$ series were used. For a further (stepwise) sample rate reduction (to $5 \mathrm{~Hz}$ ), all time series were digitally low pass filtered (FIR, $2.5 \mathrm{~Hz}$ ).

\section{RESULTS}

The entire registration of this experiment lasted 42 minutes. Only a short interval of 400 s duration was chosen for the here presented evaluation, because it exposes a spontaneously occurring phase transition. This phase transition can be identified more or less in all signals by Recurrence Plots. In Fig. 2 an example is given for the activity of the NTS neuron. The transition can be recognized here by the alteration of the texture in the recurrence plot at approximately $1180 \mathrm{~s}$. However, the transition does not occur simultaneously in all sub-systems. Three

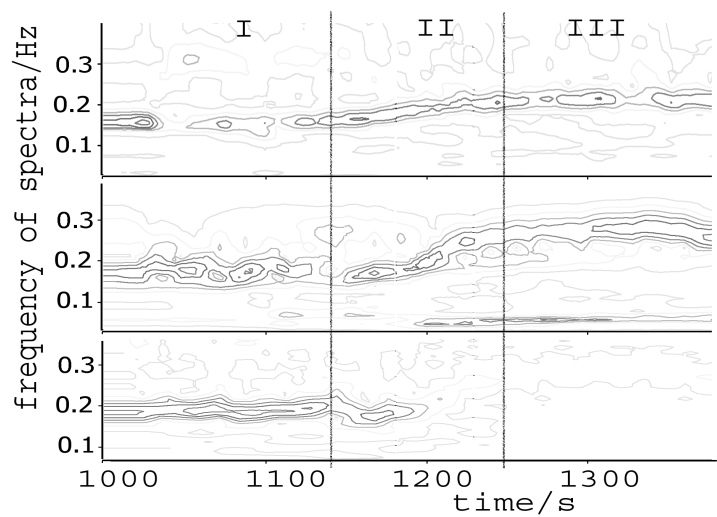

Fig. 3: Contour line plots of various Morlet wavelet spectra. (a) Time-dependent relative spectral density of the retN activity, (b) timedependent relative spectral density of IPP, (c) time-dependent amplitude spectrum of systBP. epochs can be distinguished for the temporal classification of the dynamic processes:

\section{Epoch 1000-1140s}

The first stationary epoch distinguishes itself by striking rhythmic deviations of approximately $0.18 \mathrm{~Hz}$ in the NTS neuron (ntsN), the renal sympathetic neurons (symp), and in the cardiovascular parameters (heart frequency (HF), systolic (systBP) and diastolic blood pressure (diastBP)). One of the reticular neuronal activities acquired (retN) is presented here. It shows a rhythm with a dominant frequency of $0.16 \mathrm{~Hz}$.

II. Epoch 1140-1240s

In this epoch, the phase transition actually happens. The strong rhythmicity at $0.18 \mathrm{~Hz}$ in the cardiovascular parameters $\mathrm{HF}$, systBP, diastBP as well as in the ntsN and in the symp vanishes after a temporary coordination of this rhythm (1160-1200s) with that of retN at $0.16 \mathrm{~Hz}$. Afterwards, the frequency of the rhythms increases further. The frequency of retN oscillations rises and converges with the frequency of the other reticular neuron (not shown).

III. Epoch 1240-1400s

In this epoch, again being relatively stationary, the respiratory frequency is approximately $0.28 \mathrm{~Hz}$. It is now well coordinated with the rhythms in the systBP, diastBP, HF, and in the ntsN. The amplitudes of their rhythms, however, are now strongly reduced compared to epoch I. The common rhythm in the retN's is now $0.215 \mathrm{~Hz}$.

The described transitional behavior becomes evident also by the contour line plots of various Morlet wavelet spectra of retN, IPP and systBP represented in Fig. 3.

Due to the distinct rhythmic behavior of the measured signals, the phase coordination between the different sub-systems can be well described by the differences of the instantaneous phases (Fig. 4).

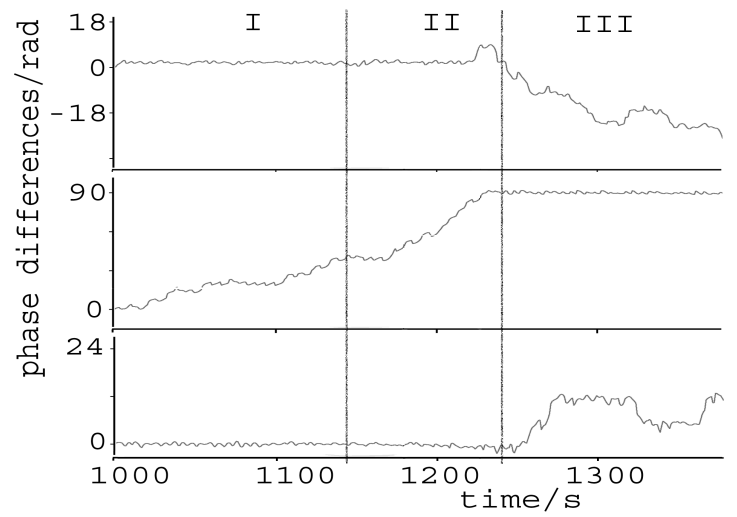

Fig. 4: Time-dependent phase differences $\Delta \Phi$ of various signals. Differences of instantaneous phases $\Delta \Phi$ are computed from pairs of signals. (a) $\Delta \Phi$ (symp, HF), (b) $\Delta \Phi$ (IPP, HF), (c) $\Delta \Phi$ (systBP, ntsN). Ordinates are scaled in radians. 

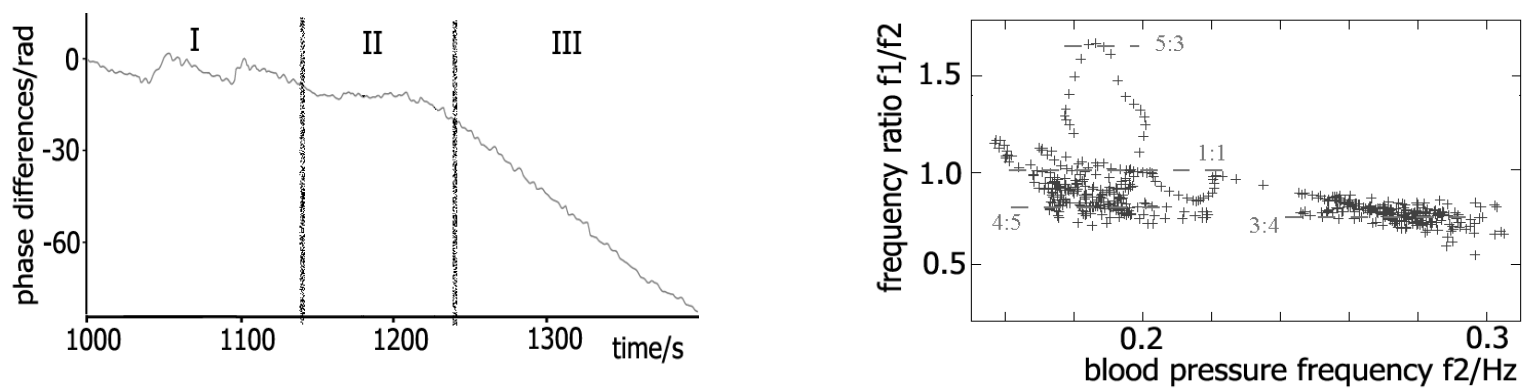

Fig. 5: Temporarily occurring synchronisation between the rhythms of systBP and retN.

(a) $\Delta \Phi$ (syst BP, retN). Up to approximately $1150 \mathrm{~s}$, the frequency relations change between 4:5 and 5:3. After that there is a 1:1 phase locking up to $1210 \mathrm{~s}$, and then a frequency relation of 3:4 is established. (b) The accompanying synchronisation diagram. Abscissa: instantaneous frequency of systBP (in the $0.05-0.5 \mathrm{~Hz}$ band), ordinate: frequency ratio f1/f2 of the rhythms of retN (f1) and systBP (f2).

Although this is not recognizable at first sight from the time-resolved spectra in Fig. 3, it becomes clear now that the rhythms of respiration and cardiovascular parameters do not show any phaselocking in epoch I, but only later in epoch III after the phase transition. This is different, e.g., in the coordination between symp and HF, or systBP and ntsN activity. Here, a phase locking occurs in epoch I, which, however, has vanished in epoch III after the phase transition. This points already to a rearrangement of the functional organization of the organism, which is characterized at first by a just slightly changing processing of baroreceptor afferents in the central nervous structures, and later by the rhythmical coordination between respiratory and cardiovascular systems.

The coordination between retN and the cardiovascular parameters (systBP, diastBP, HF), which show a phase locking merely in the transitional epoch II (1150-1210s), indicates that neurons of the RF seem to play an important role in this transition.

This can be recognized by the course of $\Delta \Phi$ (systBP, retN) in Fig. 5a. In the other epochs, the frequency relations are changing. At some points integer frequency ratios occur which results in

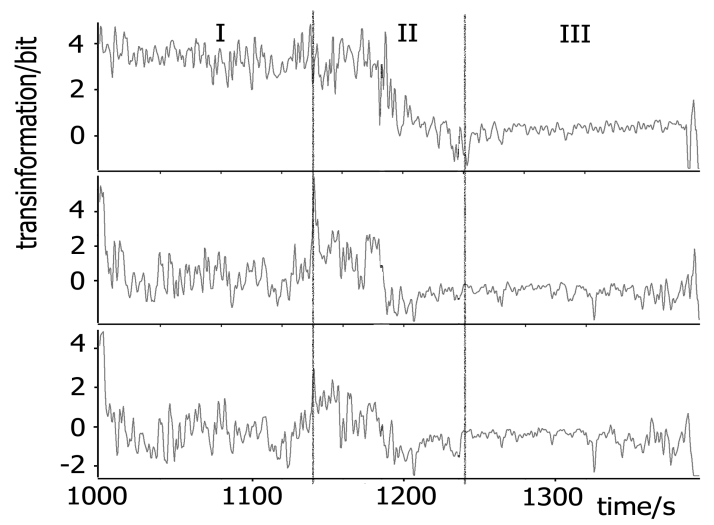

Fig. 6: Pointwise Transinformation (PTI) of time serie: (a) PTI(systBP↔ntsN) $\mathrm{m}=4, \tau=6, \mathrm{r}=0.18$; (b) $\mathrm{PTI}($ ntsN $\leftrightarrow$ retN) $\quad \mathrm{m}=5, \quad \tau=6, \quad \mathrm{r}=0.3 ; \quad$ (c) $\mathrm{PTI}($ retN $\leftrightarrow$ systBP $) \quad \mathrm{m}=5, \quad \tau=6, \quad \mathrm{r}=0.3 . \quad$ Results locally smoothed: low pass (FIR $0.5 \mathrm{~Hz}$ ). corresponding levels of the synchronization diagram of Fig. 5b. Applying PTI to our data we find before the phase transition in epoch $I$ that the $\mathrm{PTI}($ systBP $\leftrightarrow$ ntsN) shows a distinctly stronger coupling than in epoch III (Fig. 6a) which supports the above mentioned presumption that the transmission of baroreceptor afferent input is reduced at the NTS after the phase transition.

The decrease begins around 1180s and is not completely finished before $1240 \mathrm{~s}$. A similar decrease of coupling is found also in $\mathrm{PTI}($ diastBP $\leftrightarrow$ ntsN) $\quad$ and $\quad \mathrm{PTI}($ symp $\leftrightarrow \mathrm{ntsN})$ (without figure). However, this is slightly shifted in time, and in $\mathrm{PTI}(\mathrm{HF} \leftrightarrow \mathrm{ntsN})$ even a temporary increase occurs between 1210 s and 1225s. This confirms the assumption that there are different pathways for the effects of baroreceptor afferents on blood pressure and heart rate (cf. Discussion in [20]).

The coupling between reticular neurons (retN) and cardiovascular parameters and ntsN, respectively, has relatively low levels in epochs I and III, whereas it is distinctly increased in the transitional period between 1140s and 1190s (Fig. 6b,c).

(a)

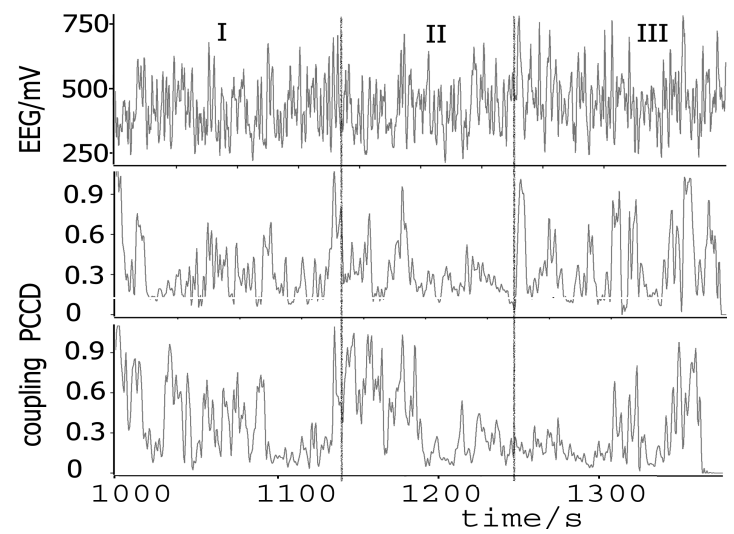

Fig. 7: Transients and couplings of EEG: (a) $\Delta(\theta-$ $\left.\mathrm{EEG}_{1}\right)$; (b) $\operatorname{PCCD}\left\{\operatorname{retN} \leftrightarrow \Delta\left(\theta-\mathrm{EEG}_{1}\right)\right\} \quad \mathrm{m}=5, \tau=6$, $\mathrm{l}=24, \mathrm{r}=0.38$; (c) $\operatorname{PCCD}\left\{\operatorname{retN} \leftrightarrow \Delta\left(\delta-\mathrm{EEG}_{\mathrm{r}}\right)\right\} \mathrm{m}=5$, $\tau=6,1=90, r=0.35$. PCCD locally smoothed: low pass (FIR, 0.5Hz). 


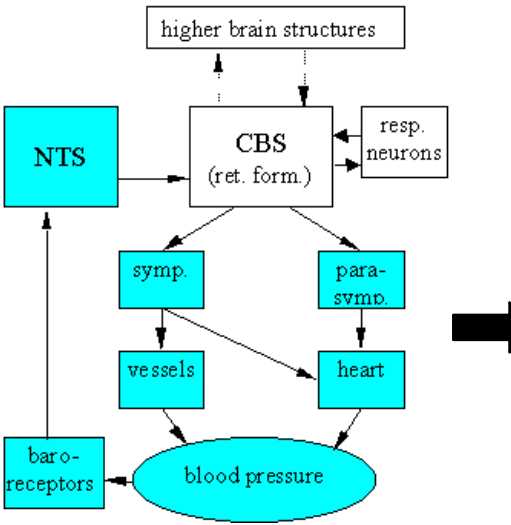

(a)

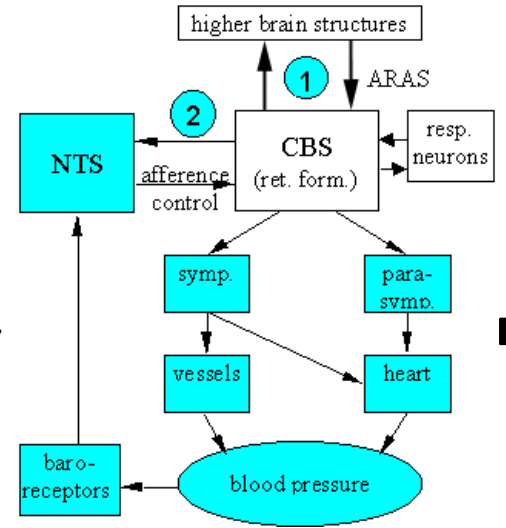

(b)

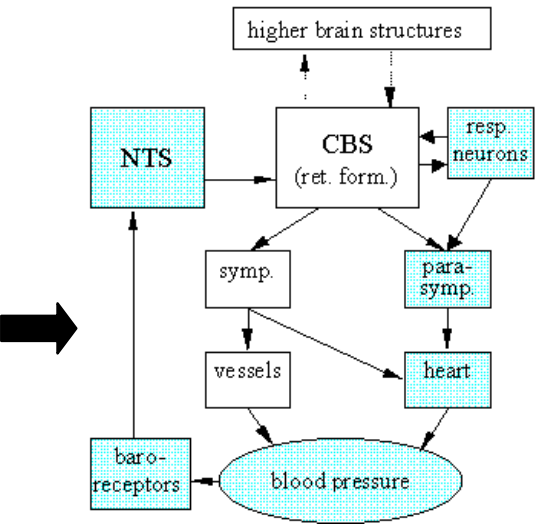

(c)

Fig. 8: Stages of the phase transition. (a) Firstly, parameters of the cardiovascular system are highly coordinated in a rhythm of about $6 \mathrm{~s}$ period duration. (b) The phase transition is initiated by mutual influences between CBS and higher brain structures (1) and a change of the processing of baroreceptor afferent input at the NTS (2). (c) After the phase transition only a weak coordination between cardiovascular and respiratory systems remains, which is indicated by the lighter boxes in (c) compared to the darker ones in (a) and (b).

This confirms the influence of the RF on the transitional process already stated above. The highest values of the $\mathrm{PTI}(\mathrm{ntsN} \leftrightarrow \mathrm{retN})$ and PTI(retN↔systBP) (Fig. 6b,c) are reached just before the beginning of epoch II at approximately 1140 s, which indicates that neurons of the RF are implicated in initiating the phase transition.

Evaluation of the EEG as an indicator of higher brain structures activities provides additional information about the mechanisms of this reorganization of the CBS. Evidence for a steep increase in coupling strength between the EEG activities and the RF at $1140 \mathrm{~s}$ is given by $\operatorname{PCCD}\left\{\right.$ retN $\leftrightarrow \Delta\left(» \theta\right.$-band», $\left.\left.\mathrm{EEG}_{1}\right)\right\}$ and $\operatorname{PCCD}\{\operatorname{retN}$ $\leftrightarrow \Delta\left(» \delta\right.$-band», $\left.\left.E G_{\mathrm{r}}\right)\right\}$ (Fig. 7b,c). This pronounced increase in coupling occurs around $0.3 \mathrm{~s}$ before the increase in coupling of $\mathrm{PTI}(\mathrm{retN} \leftrightarrow \mathrm{ntsN})$ and $\mathrm{PTI}($ retN $\leftrightarrow$ systBP), respectively. Thus, the trigger event for the phase transition may be considered as identified. Either higher brain structures signalize the beginning of the phase transition to the RF or even more probable - the CBS in the RF induces general activation in the CNS via its ascending pathways (cf. ARAS in [34], [23]).

To sum up, the dynamic course of the phase transition observed here can be represented as follows. At first, the cardiovascular parameters and the activities in the NTS and symp in epoch I are well coordinated with a marked rhythm of approximately $0.18 \mathrm{~Hz}$ (Fig. $8 \mathrm{a}$ ).

At the beginning of epoch II the phase transition is initiated by a short-time interaction between CBS and higher brain structures, probably by an activation of the CNS initiated by the CBS (or ARAS, respectively, (1) in Fig. 8b). Almost simultaneously, a strong coupling of reticular neurons to the NTS is initiated, which effects a changing in the processing of baroreceptor afferent input there ((2) in Fig. 8b). This period lasts about one minute. During this time, when actual reorganisation occurs, there is not only a strong phase coupling between CBS and NTS (in the sense of an influence on the processing of baroreceptor afferents), but also on the efferent side between CBS and cardiovascular system (Fig. 8b). This "latching" temporarily goes along with a deceleration of cardiovascular rhythms and a slight acceleration of the reticular rhythm. During the phase locking, however, the common frequency is not stable, but increases permanently.

Here, the phenomenon of "relative coordination" appears as described already by E. von Holst as typical for the transitional dynamics of rhythmically active systems [13]. A similar behavior in the rhythmic coordination of $\mathrm{CBS}$, respiration, circulation, and higher brain structures has recently been described by Lambertz and Langhorst [23].

In epoch III the re-organization has been finished. The influence of the CBS on the cardiovascular system is clearly reduced. The processing of the baroreceptor afferent input in the NTS has now been modified. This becomes obvious by the clearly reduced coupling between blood pressure and NTS activity. The sub-systems participating in the cardiovascular regulation do not expose the common "6s rhythm" any longer. Now, below $0.5 \mathrm{~Hz}$, only frequency components common with those of respiration are found, i.e. we find a coordination of respiration and cardiovascular parameters, although with considerably smaller oscillation amplitudes (Fig. 8c).

\section{DISCUSSION}

The presented results show that application of advanced tools of time series analysis can deliver useful information here concerning brainstem 
dynamics and systems states identification. We think it is noteworthy how the distinct results from the different applied algorithms support each other mutually in characterizing the phase transition and fit entirely to the results of earlier, extensive investigations.

Previous investigations in dynamic organization of the RF in the lower brainstem and its relation to peripheral and other central nervous sub-systems have been performed predominantly by linear methods, as auto- and cross-covariance functions, post-event-time histograms, amplitude and power spectra. Due to their time-averaging properties, these methods could be applied merely to stationary signal intervals. Hence, only indirect investigations of phase transitions in coordination in physiological sub-systems had been possible by concluding from results gained from sequences of relatively stable sub-epochs. These former investigations led to the concept of the CBS in the RF of the lower brainstem and the basic types of its functional organization $[4,18,19,27,31,40,41]$.

The phase transition analyzed here was taken from an experiment not used for the previous studies. However, the results confirm these former investigations on the dynamic organization of the RF in the lower brainstem and their changing relations to peripheral and other central nervous sub-systems. The special capabilities of the applied analytical methods in investigating dynamic processes is elucidated by the fact that only one single, relatively short phase transition has been necessary to confirm the results (gained by much more expense) of the investigations obtained up till now. This demonstrates that it becomes now possible to investigate systems dynamics directly as has not been possible with the methods, applied previously or merely with great experimental and temporal expenditure. Especially the finding that during phase transitions the way of processing of afferent information in the NTS is changed, is complementary to results of earlier studies obtained by means of other methods $[20,23,30,43,44]$.

Previous investigations on rhythmic coordination of different peripheral and central sub-systems [23,29] have been confirmed and complemented by these analyses as well. The rhythmic coordination between CBS neurons and the respiratory and cardiovascular system, respectively, were described as longer lasting and of other quality than the shorttime couplings between the activities of CBS neurons and the EEG [23]. The latter are short-time phenomena [23] occurring mainly when reticular rhythms in CBS neurons appear or vanish, respectively. These older results, obtained from numerous experiments, are clearly confirmed here, most of all by the PCCDs of reticular neuronal activities and the EEGs.

The more important result, however, is that the analyses yield a quasi-continuous complex description of the functional reorganization in this multi-functional system with its manifold feedback loops. To the best of the knowledge of the authors, this has not been presented in the literature before. Moreover, the phase transition analyzed here is interesting not only for demonstrating the presented methods of non-stationary time series analysis, but can also be very informative from a general physiological point of view. To what extent the analyzed phase transition is typical for reorganization processes in the CNS, and whether it might be similar to transitions between trophotropic and ergotropic functional states in general [11], will be subject of further investigations. Nevertheless, the physiological relevance of the presented phase transition is indisputable because it is not the result of external interventions but rather appeared spontaneously in the organism under physiological conditions.

\section{Acknowledgement}

The authors wish to thank Mrs. D. Starke and Mr. P. Holzner for preparing the photographs, Mrs. E. Hofmann for translating and typing the manuscript, and Mr. J. Lerch, Mr. N. Nitert, and Mr. J. Petsch for constructing essential parts of the equipment. We are also grateful to Mrs. G. Bräunig and Mrs. M. Lüdcke for their skilful technical assistance.

All computations were performed using the signal and time series analysis environment DataPlore (http://www.datan.de/DataPlore)

\section{REFERENCES}

[1] Abboud, F.M., Thames, M.D.: Interaction of cardiovascular reflexes in circulatory control. In: Handbook of Physiology, The Cardiovascular System. Peripheral Circulation. American Physiological Society, sect. 2, vol. III, part. 2, Bethesda, Maryland, (1983), 675-753.

[2] Cenys, A., Lasiene, G., Pyragas, K.: Estimation of interrelation between chaotic observables. Physica D, 52, (1991), 332-337.

[3] Cohen, L.: Time-frequency distributions - a review. Proc. IEEE, 77, (1989), 941-981.

[4] Ebinger, F., Lambertz, M. Langhorst, P.: Functional organization of the common brainstem system to different states at different times. In: Koepchen, H.P., Huopaniemi, T. (eds): Cardiorespiratory and Motor Coordination. Berlin, Heidelberg, New York, Tokyo: Springer Verlag, 1991, 160-174.

[5] Eckmann J.-P., Ruelle D., Ergodic theory of chaos and strange attractors, Rev.Mod.Physics,57(3):617-656, 1985.

[6] Eckmann J.-P., Kamphorst S. O., and Ruelle D., Recurrence plots of dynamical systems, Europhysics Letters, 4:973-979, 1987.

[7] Gabor, D.: Theory of communication. J. IEE (London), 93:429-457, 1946.

[8] Gel'fand,I.M.,Yaglom,A.M.: Calculation of the amount of information about a random function contained in another such function.Am.Math.Soc.Transl.,12:199-246,59

[9] Golenhofen, K., Hildebrandt, G.: Die Beziehungen des Blutdruckrhythmus zu Atmung und peripherer Durchblutung. PflügersArch.ges. Physiol.,267(1958)27pp.

[10] Hess, W.R.: Das physiologische Zusammenspiel von Kreislauf und Atmung. Verh. Dtsch. Ges. Kreislaufforsch., 8. Tagung (1935). 
[11] Hess, W.R., Das Zwischenhirn, 2. Auflage, Benno Schwabe, Basel, 1954, 218 pp.

[12] Hildebrandt, G.: Zur Frage der Rhythmischen Funktionsordnung beim Menschen. In: Delius, L., Koepchen, H.P., Witzleb, E. (eds.): Probleme der zentralnervösen Regulationen, Bad Oeynhausener Gespräche V. Berlin: Springer-Verlag 1962.

[13] Holst, E. von, Die relative Koordination als Phänomen and als Methode zentralnervöser Funktionsanalysen, Erg. Physiol., 42 (1939) 228-306.

[14] Koch E., Die Irradiation der pressorischen Kreislaufreflexe, Klin. Wschr. 2, (1932), 225-227.

[15] Koepchen, H.P.: Die Blutdruckrhythmik. Darmstadt: Dr. Dietrich Steinkopff Verlag, 1962.

[16] Koepchen, H.P.: Physiology of rhythms and control systems. An integrative approach. In: Haken, H., Koepchen, H.P. (eds.): Springer Series in Synergetics, vol. 55, Rhythms in Physiological Systems. Berlin, Heidelberg: Springer-Verlag 1991, 3-20.

[17] Korner, P.I.: Central nervous control of autonomic cardiovascular function. In: Handbook of Physiology. The Cardiovascular System. The Heart. American Physiological Society, sect.2, vol. I, Bethesda, Maryland, (1979), 691-739.

[18] Lambertz, M., Schulz, G. and Langhorst, P., Reticular formation of the lower brainstem. A common system for cardio-respiratory and somatomotor functions. Considerations aided by computer simulations, J. Auton. Nerv. Syst., 12 (1985) 63-75.

[19] Lambertz,M., Kluge,W., Schulz, G. and Langhorst, P., Principles of functional organization of a common system in the reticular formation for cardiorespiratory and somatomotor regulation. Computer simulations based on physiological data, J.Auton.Nerv.Syst.(Suppl.)(1986)pp.269-274.

[20] Lambertz, M., Kluge, W. and Langhorst, P., Discharge patterns of neurons in the nucleus tractus solitarii (NTS): Its cardiac rhythm is modulated by firing rate of the neurons, J. Auton. Nerv. Syst., 44 (1993) 137-150

[21] Lambertz, M. and Langhorst, P., Cardiac rhythmic patterns in neuronal activity are related to the firing rate of the neurons: I. Brainstem reticular neurons of dogs, J. Auton. Nerv. Syst., 51 (1995) 153-163.

[22] Lambertz, M., Schulz, G. and Langhorst, P., Cardiac rhythmic patterns in neuronal activity related to the firing rate of the neurons: II. Amygdala neurons of cats, J. Auton. Nerv. Syst., 51 (1995) 165-173.

[23] Lambertz M. and Langhorst P., Simultaneous changes of rhythmic organization in brainstem neurons, respiration, cardiovascular system and EEG between $0.05 \mathrm{~Hz}$ and 0.5 Hz, J. Auton. Nerv. Syst., 68 (1998) 58-77.

[24] Langhorst, P., Stroh-Werz, M., Dittmar, K., Camerer, H.: Facultative coupling of reticular neuronal activity with peripheral cardiovascular and central cortical rhythms Brain Res. 87, (1975), 407-418.

[25] Langhorst, P., Schulz, B., Lambertz, M., Schulz, G., Camerer, H.: Dynamic characteristics of the "unspecific brain stem system". In: Koepchen, H.P., Hilton, S.M., Trzebski, A. (eds.): Central Interaction Between Respiratory and Cardiovascular Control Systems. Berlin, Heidelberg, New York: Springer-Verlag, 1980, pp. 30-41.

[26] Langhorst, P., Lambertz, M., Schulz, G.: Central control and interactions affecting sympathetic and parasympathetic activity. J.Auton.Nerv.Syst.4,(1981), 149-163.

[27] Langhorst,P.,Schulz,B.,Schulz,G.,Lambertz, M.,with technical assistance of Krienke, B.: Reticular formation of the lower brainstem. A common system for cardiorespiratory and somatomotor functions: discharge patterns of neighboring neurons influenced by cardiovascular and respiratory afferents. J.Auton.Nerv.Syst.9, (1983), 411-432.

[28] Langhorst, P., Schulz, G., Lambertz, M.: Oscillating neuronal network of the "common brain stem system". In: Miyakawa, K., Koepchen, H.P., Polosa, C. (eds.): Mechanisms of Blood Pressure Waves. Tokyo/Berlin: Japan Sci. Soc. Press/Springer-Verlag, 1984, pp. 257-275.
[29] Langhorst, P., Lambertz, M., Schulz, G.: Assessment of rhythmicity in the visceral nervous system. In: Lown, B., Malliani, A., Prosdocimi, M. (eds.): Neuronal Mechanisms and Cardiovascular Disease. Padova: Liviana Press/Springer-Verlag, Fidia Research Series, vol. 5, (1986), pp. 133-144.

[30] Langhorst,P.,Lambertz,M.,Kluge,W.and RittwegerJ., Different modes of dampening influence from baroreceptors are determined by the functional organization of the NTS neuronal network,J.Auton.Nerv.Syst.,41,141-156,92.

[31] Langhorst, P., Schulz, B.G., Seller, H. and Koepchen, H.P., Convergence of visceral and somatic afferents on single neurons in the reticular formation of the lower brain stem in dogs, J. Auton. Nerv. Syst., 57 (1996) 149-157.

[32] Learned, R.E., Willsky, A.S.: A wavelet packet approach to transient signal classification. Tech.Rep.LIDSP-2199, Lab.Inf.Decis.Sys.,MassachusettsInst.Techn.,93

[33] Morlet, J.,Arehs,G., Fourgeau, I.,Giard,D.: Wave propagation and sampling theory.Geophysics,47:203-236, 82

[34] Moruzzi, G., The sleep-waking cycle. In R.H. Adrian et al (Eds.), Ergebnisse der Physiologie, Reviews of Physiology,Biochemistry and Experimental Pharmacology, Vol 64, Springer Verlag, Berlin (1972) pp. 1-165.

[35] Pompeiano, O.: Reticular Formation. In: Iggo, A. (ed.): Handbook of Sensory Physiology, vol. 2. Somatosensory System. Berlin: Springer-Verlag, (1973), 381-488.

[36] Raschke, F.: Die Kopplung zwischen Herzschlag und Atmung beim Menschen.Thesis,Universität Marburg,1981.

[37] Rassler, B., Waurick, S., Ebert, D.: Einfluss zentralnervöser Koordination im Sinne v. HOLSTs auf die Steuerung von Atem- und Extremitätenmotorik des Menschen. Biol. Cybern., 63 (1990) 457-462.

[38] Rihaczek, A.W.: Hilbert transforms and the complex representation of real signals.Proc.IEEE,54:434-435, 1966.

[39] Scheibel, A.B.: The brain stem reticular core and sensory function. In: Handbook of Physiology. The Nervous System, vol. III, Sensory Processes, part 1. American Physiological Society, Bethesda, Maryland, (1984), 213-256.

[40] Schulz, B., Lambertz, M., Schulz, G., Langhorst, P., with technical assistance of Krienke, B.: Reticular formation of the lower brainstem. A common system for cardiorespiratory and somatomotor functions: discharge patterns of neighboring neurons influenced by somatosensory afferents. J. Auton. Nerv. Syst. 9, (1983), 433-449.

[41] Schulz,G.,Lambertz,M.,Schulz,B.,Langhorst,P,Krienke , B.: Reticular formation of the lower brain stem. A common system for cardiorespiratory and somatomotor functions. Cross-correlation analysis of discharge patterns of neighboring neurons.J.Auton.Nerv.Syst.12,(1985),35-62

[42] Shannon, C.E., Weaver, W.: The Mathematical Theory of Communication. The University of Illinois Press, Urbana, 1949.

[43] Stroh-Werz,M.,Langhorst,P., Camerer, H., Neuronal activity with cardiac rhythm in the nucleus of the solitary tract in cats and dogs. I. Different discharge patterns related to the cardiac cycle, Brain Res., 133 (1977) 65-80.

[44] Stroh-Werz,M.,Langhorst,P., Camerer,H., Neuronal activity with cardiac rhythm in the nucleus of the solitary tract in cats and dogs. II. Activity modulation in relation to the respiratory cycle, BrainRes.,133(1977) 81-93

[45] Takens, F.: Detecting strange attractors in turbulence. In: Rand, D., Young, L.S. (eds.): Dynamical Systems and Turbulence, Warwick 1980, 366-381, Springer-Verlag, Berlin, 1981, Lecture Notes in Mathematics, p. 898.

[46] Tegtmeier, H.: Verfahren zur Rauschunterdrückung und Transientendetektion bei physiologischen Zeitreihen basierend auf der Wavelet-Transformation und der nichtlinearen Dynamik. Diplomarbeit, Institut für Physiologie, RWTH Aachen, 1997.

[47] Vandenhouten R., Analyse instationärer Zeitreihen komplexer Systeme und Anwendungen in der Physiologie, Diss. RWTH Aachen, Shaker, 1998. 